\title{
Electron screening in Al and Ni metals
}

\author{
Jelena Gajević* \\ Jožef Stefan Institute, Ljubljana, Slovenia \\ E-mail: jelena.gajevic@ijs.si

\section{Miha Škof} \\ Faculty of Mathematics and Physics, Ljubljana, Slovenia
}

\section{Primož Pelicon}

Jožef Stefan Institute, Ljubljana, Slovenia

\section{Matej Lipoglavšek}

Jožef Stefan Institute, Ljubljana, Slovenia

\begin{abstract}
Proton induced nuclear reactions were studied over the bombarding energy range from 0.98 to 3.15 MeV for different environments: $\mathrm{Ni}$ and $\mathrm{Al}$ metals, $\mathrm{Al}_{2} \mathrm{O}_{3}$ and $\mathrm{NiO}$ insulators. The measurements were based on observation of the yields of ${ }^{59,61,63,64,65} \mathrm{Cu},{ }^{58,60,62} \mathrm{Ni}$ and ${ }^{28} \mathrm{Si}$ de-excitation $\gamma$ rays. The resonances: ${ }^{58} \mathrm{Ni}(\mathrm{p}, \gamma){ }^{59} \mathrm{Cu},{ }^{58} \mathrm{Ni}\left(\mathrm{p}, \mathrm{p}^{\prime} \gamma\right){ }^{58} \mathrm{Ni}$ and ${ }^{27} \mathrm{Al}(\mathrm{p}, \gamma){ }^{28} \mathrm{Si}$ were measured. Shifts in resonance energy for the metalic target relative to the insulator ones were not observed. Large electron screening was seen only in the ${ }^{64} \mathrm{Ni}(\mathrm{p}, \mathrm{n} \gamma){ }^{64} \mathrm{Cu}$ reaction.
\end{abstract}

VI European Summer School on Experimental Nuclear Astrophysics

September 18-27, 2011

Hotel S.Tecla Palace, Acireale, Italy

${ }^{*}$ Speaker. 


\section{Introduction}

Due to Coulomb repulsion, the cross-section $\sigma$ for charged-particle-induced nuclear reactions drops rapidly with decreasing beam energy. The astrophysical $S$-factor is usually introduced to separate the strong energy dependence from effects of pure nuclear interactions [1]. The cross section $\sigma$ is then written as a function of the c.m.s energy $E$ as:

$$
\sigma(E)=\frac{S(E)}{E} e^{-2 \pi \eta}
$$

where $\eta=Z_{1} Z_{2} e^{2} / 4 \pi \varepsilon_{0} \hbar \sqrt{2 E / \mu}$ is the Sommerfeld parameter, $Z_{1}$ and $Z_{2}$ are the charge numbers of interacting nuclei and $\mu$ their reduced mass. In this way, all nuclear interactions are described by $S(E)$, which in case of non-resonant reactions varie slowly with energy while the exponential Gamow factor $e^{-2 \pi \eta}$ describes the s-wave penetration through the Coulomb barrier of point-like charges and thus accounts for strong energy dependence of the cross sections at sub-Coulomb barrier energies. It is known that the cross-section increases at low energies when the interacting nuclei are not bare but embedded in the electron cloud of an atom [1]. The enhancement factor, $f$, could be written as:

$$
f(E)=\frac{\sigma\left(E+U_{e}\right)}{\sigma(E)}
$$

Experimental studies of different nuclear reactions (see for instance [2] - [4]) have shown the expected enhancement at low energies, corresponding to a screening potentials $U_{e}$ significantly larger than theoretically expected. It was observed that the magnitude of the screening effect strongly depends on the host material and the reason for this dependence is not precisely known. In addition, an increase of the screening potential proportional to proton number $Z_{2}$ of the target was observed in $\mathrm{Li}, \mathrm{Be}, \mathrm{V}$ and Lu targets [3], [5]. Also, shifts in resonance energy for Lu metallic targets relative to the insulator ones were observed.

\section{Experiment}

To further investigate the electron screening effects at high $Z$, we studied proton induced nuclear reactions induced on different environments; $\mathrm{Ni}$ and $\mathrm{Al}$ metals, $\mathrm{Al}_{2} \mathrm{O}_{3}$ and $\mathrm{NiO}$ insulators. Proton beams with energies between 0.98 and $3.15 \mathrm{MeV}$ were accelerated by the $2 \mathrm{MV}$ Tandetron accelerator at Jožef Stefan Institute. The measurements were based on observation of the yields of ${ }^{59,61,63,64,65} \mathrm{Cu},{ }^{58,60,62} \mathrm{Ni}$ and ${ }^{28} \mathrm{Si}$ de-excitation $\gamma$ rays, populated in the reactions ${ }^{58} \mathrm{Ni}(\mathrm{p}, \gamma)^{59} \mathrm{Cu}$, ${ }^{60} \mathrm{Ni}(\mathrm{p}, \gamma){ }^{61} \mathrm{Cu}, \quad{ }^{62} \mathrm{Ni}(\mathrm{p}, \gamma){ }^{63} \mathrm{Cu}, \quad{ }^{64} \mathrm{Ni}(\mathrm{p}, \gamma){ }^{65} \mathrm{Cu} \quad$ and $\quad{ }^{58} \mathrm{Ni}\left(\mathrm{p}, \mathrm{p}^{\prime} \gamma\right){ }^{58} \mathrm{Ni}, \quad{ }^{60} \mathrm{Ni}\left(\mathrm{p}, \mathrm{p}^{\prime} \gamma\right){ }^{60} \mathrm{Ni}$, ${ }^{62} \mathrm{Ni}\left(\mathrm{p}, \mathrm{p}^{\prime} \gamma\right){ }^{62} \mathrm{Ni},{ }^{64} \mathrm{Ni}(\mathrm{p}, \mathrm{n} \gamma){ }^{64} \mathrm{Cu}$ and ${ }^{27} \mathrm{Al}(\mathrm{p}, \gamma){ }^{28} \mathrm{Si}$. All the reactions, except ${ }^{64} \mathrm{Ni}(\mathrm{p}, \mathrm{n}){ }^{64} \mathrm{Cu}(Q=$ $-2457.381 \mathrm{keV})$, have positive $Q$ values. Gamma rays were detected with a high purity germanium (HPGe) detector positioned $5 \mathrm{~cm}$ from the target at an angle of $60^{\circ}$ wih respect to the proton beam direction. Proton dose was deduced from the peak area in the Rutherford back-scattering (RBS) spectrum pertaining to protons which are backscattered from a beam chopper. A thin Au foil is deposited on the surface of a rotating graphite chopper which intersects the proton beam with a frequency of $10 \mathrm{~Hz}$ [6]. Backscattered protons were detected with a passivated implanted planar silicon (PIPS) detector. The beam current was of the order of $90 \mathrm{nA}$. The natural Ni target with $99.98 \%$ purity obtained from Goodfellow was $100 \mu \mathrm{m}$ thick. The thickness of the Al target, from 


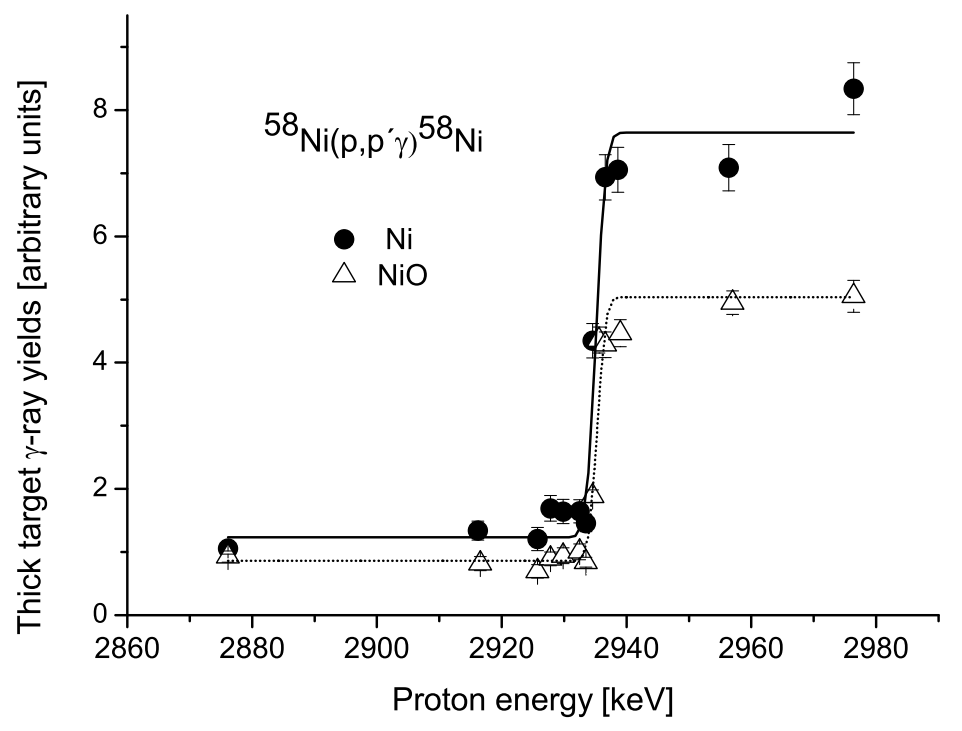

Figure 1: Thick target $1454 \mathrm{keV} \gamma$-ray yields from the ${ }^{58} \mathrm{Ni}\left(\mathrm{p}, \mathrm{p}^{\prime} \gamma\right)^{58} \mathrm{Ni}$ reaction near the $E_{p}=2935 \mathrm{keV}$ resonance. Lines are fits to the data (see text).

which the $\mathrm{Al}_{2} \mathrm{O}_{3}$ layer has been removed before starting the measurements, was $1 \mathrm{~mm}$. NiO powder with $99.995 \%$ purity was obtained from Chempur. A $1 \mathrm{~mm}$ thick $\mathrm{NiO}$ target was prepared by pressing the $\mathrm{NiO}$ powder, while $\mathrm{Al}_{2} \mathrm{O}_{3}$ target was prepared by heating $\mathrm{Al}\left(\mathrm{OH}_{3}\right)$ on tantalum substrate. The resonances ${ }^{58} \mathrm{Ni}(\mathrm{p}, \gamma){ }^{59} \mathrm{Cu}$ at proton energy $E_{p}=1424 \mathrm{keV}$ and $1844 \mathrm{keV},{ }^{58} \mathrm{Ni}\left(\mathrm{p}, \mathrm{p}^{\prime}\right)^{58} \mathrm{Ni}$ at $E_{p}=2935 \mathrm{keV}$ (all energies obtained from [8]) and ${ }^{27} \mathrm{Al}(\mathrm{p}, \gamma){ }^{28} \mathrm{Si}$ at $E_{p}=992 \mathrm{keV}$ [9] were measured. We chose these $\mathrm{Ni}$ resonances because they were the strongest in our energy range and the well known $E_{p}=992 \mathrm{keV}{ }^{27} \mathrm{Al}(\mathrm{p}, \gamma)^{28} \mathrm{Si}$ [8] resonance to reexamine a previous accelerator energy calibration. Thick target $1454 \mathrm{keV} \gamma$-ray yields produced in deexcitation of first excited state in ${ }^{58} \mathrm{Ni}$ from the ${ }^{58} \mathrm{Ni}\left(\mathrm{p}, \mathrm{p}^{\prime} \gamma\right){ }^{58} \mathrm{Ni}$ reaction near the $E_{p}=2935 \mathrm{keV}$ resonance as a function of laboratory beam energy are shown in fig. 1 for $\mathrm{Ni}$ metal and $\mathrm{NiO}$ insulator. Thick target $1779 \mathrm{keV} \gamma$-ray yields produced in deexcitation of ${ }^{28} \mathrm{Si}$ from the ${ }^{27} \mathrm{Al}(\mathrm{p}, \gamma){ }^{28} \mathrm{Si}$ reaction near the $E_{p}=992 \mathrm{keV}$ resonance are shown in fig. 2 for $\mathrm{Al}$ metal and $\mathrm{Al}_{2} \mathrm{O}_{3}$ insulator.

\section{Results}

We fitted the thick target $\gamma$-ray yield $N_{\gamma}$ near resonance energy $E_{r}$ by using the equation:

$$
N_{\gamma}=A+B \cdot \operatorname{erf}\left(\frac{E_{p}-E_{r}}{\sqrt{2} \Gamma}\right)
$$

where the parameter A is the mean value, B is the resonance height and $\operatorname{erf}(x)=\frac{2}{\sqrt{\pi}} \int e^{-x^{2}} \mathrm{~d} x$. From the observed thick target $\gamma$-ray yields near all the studied resonances in $\mathrm{Ni}$ and $\mathrm{Al}$, we didn't notice any shifts in resonance energy for the metallic targets relative to the insulator ones, furthermore the values were the same within experimental errors of about $2 \mathrm{keV}$. We observed no resonance shifts 


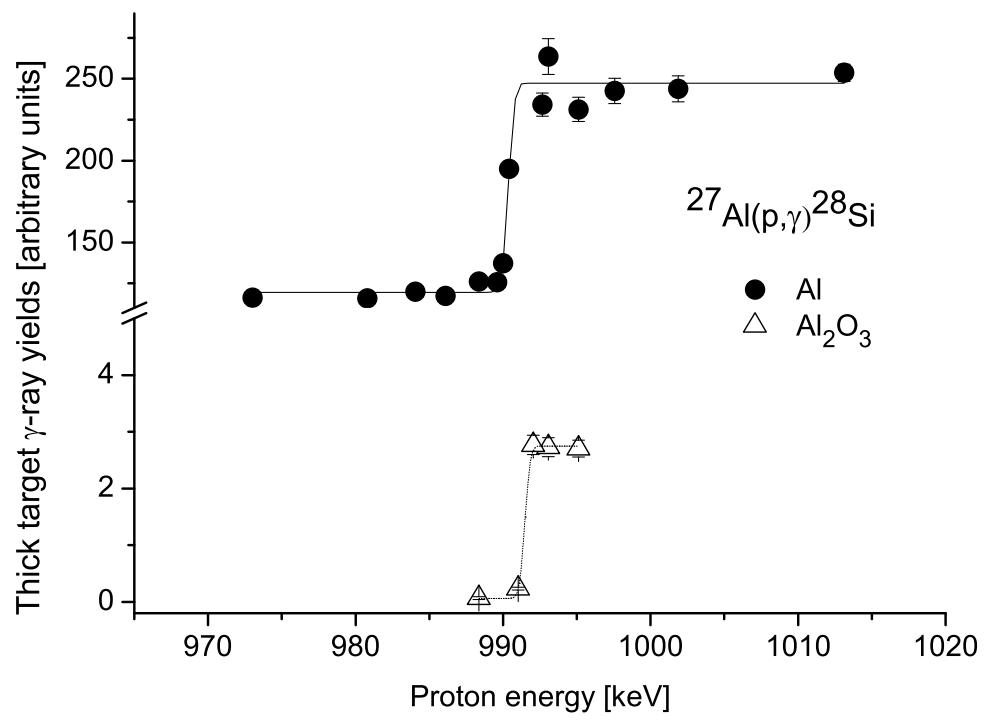

Figure 2: Thick target $1779 \mathrm{keV} \gamma$-ray yields from the ${ }^{27} \mathrm{Al}(\mathrm{p}, \gamma)^{28} \mathrm{Si}$ reaction near the $E_{p}=992 \mathrm{keV}$ resonance. Lines are fits to the data (see text).

in our reaction and consequently no large electron screening. The height of resonance fit is equal to the integrated cross section, which is nearly equal to the product of the resonance width and height. Since cross-section strongly depends on the Coulomb barrier effect (at sub-Coulomb energies), one might expect noticable difference in resonance heights at low energies. In resonant ${ }^{58} \mathrm{Ni}\left(\mathrm{p}, \mathrm{p}^{\prime} \gamma\right){ }^{58} \mathrm{Ni}$ reaction the proton must penetrate Coulomb barrier two times, firstly in the entrance channel it will penetrate the Coulomb barrier of ${ }^{58} \mathrm{Ni}$ (with incident energy $E_{p}$ ) forming a compound ${ }^{59} \mathrm{Cu}$ nucleus which will consequently decay by emitting a proton and a $\gamma$ ray. In order to leave the ${ }^{59} \mathrm{Cu}$ nucleus, the proton has to again penetrate Coulomb barrier (with energy $E_{p}-E_{\gamma}$ ). Therefore, we expected a large difference in resonance heights for metalic and insulator target. The heights of resonance at $E_{p}=2935 \mathrm{keV}$ populating the first excited state in ${ }^{58} \mathrm{Ni}$ at $1454 \mathrm{keV}$ differed only by the differences in stoichiometry and stopping power for nickel metal and insulator targets, implying no large electron screening in this reaction. Results were the same for resonances in ${ }^{58} \mathrm{Ni}(\mathrm{p}, \gamma){ }^{59} \mathrm{Cu}$ reaction at $E_{p}=1424 \mathrm{keV}$ and $E_{p}=1844 \mathrm{keV}$. On the other hand, results obtained for population of the first excited state in ${ }^{64} \mathrm{Cu}$ at $159 \mathrm{keV}$ [7] produced in the ${ }^{64} \mathrm{Ni}(\mathrm{p}, \mathrm{n} \gamma){ }^{64} \mathrm{Cu}$ reaction show significantly higher cross section for the metallic nickel compared to the insulator target indicating a large electron screening.

The measured number of $\gamma$ rays, $N_{\gamma}$ in the case of a thin target is according to the definition of the cross section:

$$
N_{\gamma}=\varepsilon N_{p} \frac{\rho N_{A} x}{M} \sigma
$$

where $\varepsilon$ is the $\gamma$-ray detection efficiency, $N_{p}$ the number of incident protons, $N_{A}$ Avogardo's number and $\sigma, \mathrm{x}$, and $\mathrm{M}$ the density, thickness and molar mass of the target, respectively. The thick target proton particle yields had to be calculated by transforming eq.(3.2) into a differential form and 
integrating over energies from threshold energy $E_{t h}$ to the beam energy $E_{p}$ :

$$
N_{\gamma}=\varepsilon N_{p} \frac{\rho N_{A}}{M} \int_{E_{t h}}^{E_{p}} \frac{\sigma(E)}{\mathrm{d} E_{0} / \mathrm{d} x} \mathrm{~d} E_{0}
$$

The stopping power $\mathrm{dE}_{0} / \mathrm{dx}$ was calculated using SRIM [10]. Gamma ray efficiency was determined using a calibrated $\mathrm{Eu}^{152}$ source. We assumed that there is no electron screening in $\mathrm{NiO}$ [2] and took the cross section for populating the $159 \mathrm{keV}$ state in ${ }^{64} \mathrm{Cu}$ from ref. [11] from threshold energy at $2.66 \mathrm{MeV}$ to $2.71 \mathrm{MeV}$. Above this energy the cross section from ref. [11] did not fit our data well. Therefore, we took the cross section for the ${ }^{64} \mathrm{Ni}(\mathrm{p}, \mathrm{n}){ }^{64} \mathrm{Cu}$ reaction from [12] from $2.71 \mathrm{MeV}$ to the end of our energy range. We scaled this cross section to make the combined cross section continuous at $2.71 \mathrm{MeV}$. With the combined cross section we fitted our data on $\mathrm{NiO}$ target and got good agreement (see fig.3). We compared the $\gamma$-ray yields of metallic target with the $\gamma$-ray yields of the insulator for all the studied $\gamma$ rays in $(\mathrm{p}, \gamma)$ and $\left(\mathrm{p}, \mathrm{p}^{\prime} \gamma\right)$ reactions. An average value $\alpha=N_{\gamma}^{\mathrm{Ni}} / N_{\gamma}^{\mathrm{NiO}}$ has been then determined. In this way, we got yield enhancement factor, $\alpha$, not influenced by electron screening. The energy dependence of the stopping powers [10] of Ni and $\mathrm{NiO}$ is identical within $1 \%$ in our energy range leading to a constant $\alpha=1.42$ because of the different stoichiometries. However, we must stress that our fitted $\alpha$ was slightly larger than we expected, $\alpha=1.510(9)$. A similar difference was also observed in ref. [5] for $\mathrm{V}$ and $\mathrm{VO}_{2}$. Due to the the reaction threshold, the electron screening could not be taken into account in the usual way by replacing $\sigma(E)$ with $\sigma\left(E+U_{e}\right)$. Instead, the electron screening was taken into account by replacing $\sigma(E)$ in the case of the insulator with $p\left(U_{e}\right) \sigma(E)$ in the case of metallic target where $p\left(U_{e}\right)$ is the ratio of Coulomb barrier pentrabilities (given by Gamow factors) for metalic nickel and nickel oxide:

$$
p\left(U_{e}\right)=\frac{e^{\left(-2 \pi \eta\left(E+U_{e}\right)\right)}}{e^{(-2 \pi \eta(E))}}=\frac{e^{\left(-2 \pi \alpha Z_{1} Z_{2} c \sqrt{\frac{\mu}{2\left(E+U_{e}\right)}}\right)}}{e^{\left(-2 \pi \alpha Z_{1} Z_{2} c \sqrt{\frac{\mu}{2 E}}\right)}}
$$

Using Taylor expansion for $U_{e} / E \ll 1$ we get:

$$
p\left(U_{e}\right)=e^{\pi \alpha Z_{1} Z_{2} \sqrt{\frac{\mu c^{2}}{2 E}} \cdot U_{e} / E}
$$

The electron screening potential was fitted to the data using $N_{\gamma}^{\mathrm{Ni}}=\alpha N_{\gamma}^{\mathrm{NiO}}\left(E+U_{e}\right)$. Experimentally obtained $\alpha$ was used for fitting. A value of $U_{e}=44 \mathrm{keV}$ was obtained from one-parameter leastsqares fit to the data. Statistical error of the fit is $2.9 \mathrm{keV}$ while systematic error due to uncertainty in the experimentaly obtained $\alpha$ is $2.1 \mathrm{keV}$ and due to uncertainty of self absorption of the 159 $\mathrm{keV} \gamma$-ray in the target is $17.3 \mathrm{keV}$ giving the systematic error of $17.4 \mathrm{keV}$. The combined error is $18 \mathrm{keV}$. Additional sources of systematic error might be found in $\varepsilon$ and the cross section for ${ }^{64} \mathrm{Ni}(\mathrm{p}, \mathrm{n} \gamma){ }^{64} \mathrm{Cu}$ reaction but these are much smaller than above quoted errors. It must be stressed that we have not determined $U_{e}$ by the above procedure, since we assumed no screening in NiO. Instead we measured $U_{D}=U_{e}-U_{A}$, which is the difference between screening potentials in Ni and NiO. For further explanation see ref. [5]. The results are shown in fig. 3 for Ni metal and $\mathrm{NiO}$ insulator, where the solid curve represents the fit due to $U_{D}=44 \mathrm{keV}$ for $\mathrm{Ni}$ and dotted curve the fit with $U_{A}=0 \mathrm{keV}$ for $\mathrm{NiO}$. 


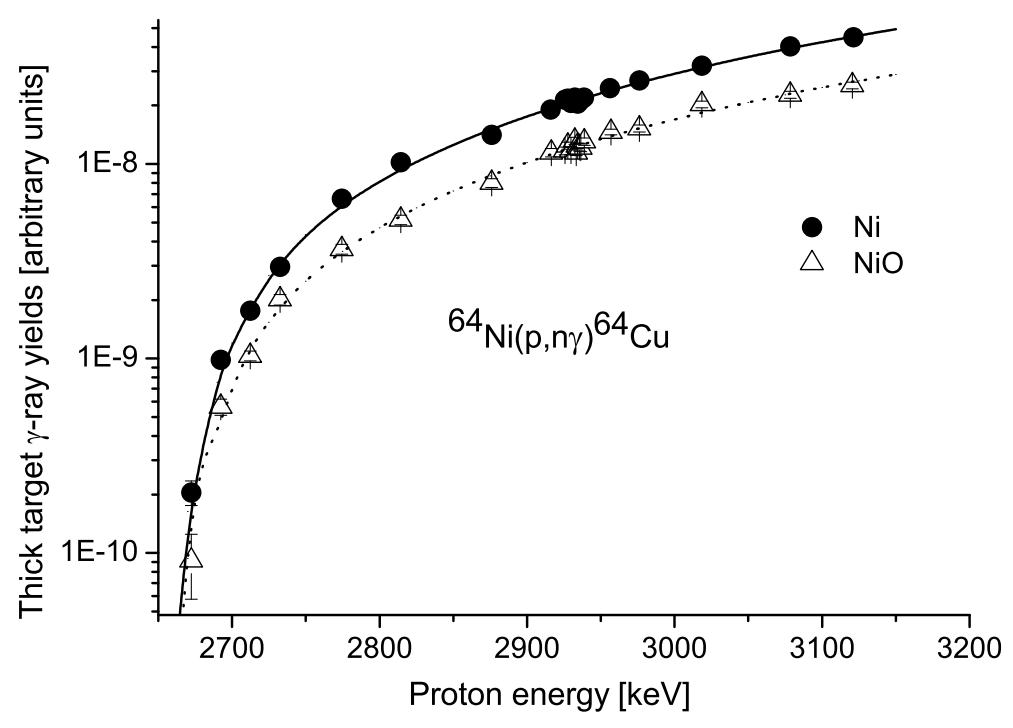

Figure 3: Thick target $\gamma$-ray yields of the $159 \mathrm{keV} \gamma$ ray from ${ }^{64} \mathrm{Ni}(\mathrm{p}, \mathrm{n} \gamma){ }^{64} \mathrm{Cu}$ reaction for $\mathrm{Ni}$ and $\mathrm{NiO}$ targets. Lines are fits to the data.

\section{Conclusions}

Kettner et al. [5] observed a narrow resonance in the ${ }^{176} \mathrm{Lu}(\mathrm{p}, \mathrm{n}){ }^{176} \mathrm{Hf}$ reaction at $E_{p}=810 \mathrm{keV}$ and reported lowering of this resonance energy by $U_{D}=32 \pm 2 \mathrm{keV}$ and $U_{D}=33 \pm 2 \mathrm{keV}$ for the $\mathrm{Lu}$ metal and $\mathrm{PdV}_{10 \%}$ alloy respectively, relative to the insulator. The sizable resonance shifts were interpreted as a demonstration of the acceleration effect by the valence electrons.

We have not observed shifts in resonance energy for metallic targets relative to insulator ones neither for $\mathrm{Al}$ nor $\mathrm{Ni}$ target. Our results seem to indicate that large electron screening is present only in $(\mathrm{p}, \mathrm{n})$ reactions and not in the relatively slower $(\mathrm{p}, \gamma)$ and $\left(\mathrm{p}, \mathrm{p}^{\prime} \gamma\right)$ reactions. The main difference between these reactions is the time scale on which they occur. While a neutron can be emitted from a compound nucleus relatively quickly, (comparable to the reaction time of the order $10^{-22} s$ ) the half lives of the resonances studied are several orders of magnitude larger, about $10^{-17} s$ [8]. In a very similar $(\mathrm{p}, \mathrm{n})$ reaction in vanadium, which has only three protons less than nickel, an electron screening potential of $U_{D}=27 \pm 9 \mathrm{keV}$ was measured [5]. Due to the prediction [5] of the linear dependance of $U_{D}$ on target $\mathrm{Z}$, we expected $U_{D}$ in Ni to be around $30 \mathrm{keV}$. Our expectation was corroborated by the measurement of $U_{D}=32 \pm 2 \mathrm{keV}$ in lutetium. Suprisingly, our result of $U_{D}=44 \pm 18 \mathrm{keV}$ is substantially higher than expected. Although the large error bars still allow $U_{D}$ to be the same in $\mathrm{Ni}$ and $\mathrm{V}$, our screening potential for $\mathrm{Ni}$ is actually the highest screening potential ever measured. Due to its suprising nature, our result will require a lot of scrutiny in the future, but it anyway points to a conclusion that electron screening dependence on $Z$ is not simply linear. It seems that electron screening depends on the target material in a more complicated way, similar to the case of implanted deuterons and the $d(d, p) t$ reaction. 


\section{References}

[1] H. J. Assenbaum, K. Langanke, C. Rolfs, Z. Phys. A 327, (1987) 461.

[2] C. Rolfs, Prog. Theor. Phys. Suppl 154, (2004) 373.

[3] J. Cruz et al., Phys. Lett. B 624, (2005) 181.

[4] J. Kasagi, Prog. Theor. Phys. Suppl. 154, (2004) 365.

[5] K. U. Kettner et al., J.Phys G 32, (2006) 489.

[6] K. Vogel-Mikuš et al., Nucl. Instr. and Meth. B 267, (2009) 2884.

[7] Nuclear Data Sheets 108, 197 (2007).

[8] Nuclear Data Sheets 95, 215 (2002).

[9] M. J. F. Healy., Nucl. Instr. and Meth. B, 249, (2006) 918.

[10] J. F. Ziegler, J. P. Biersack and M. D. Zeigler, The Stopping and range of Ions in matter, Lulu press Co.; Morrisville, NC, 2008; http://www.srim.org

[11] B. A. Nemashkalo et al., Yadernaya Fizika, 37 (1983) 3. Data taken from the EXFOR database, file EXFOR A0112.001, retrieved from the IAEA Nuclear Data Services website.

[12] B. Ja. Guzhovskij et al., Izv. Rossiiskoi Akademii Nauk, Ser. Fiz. 33 (1969) 129. Data taken from the EXFOR database, file EXFOR F0704.001 retrieved from the IAEA Nuclear Data Services website. 